\title{
Atmospheric Eddies Mediate Lapse Rate Feedback and Arctic Amplification $\mathscr{O}$
}

\author{
NICOLE FELDL \\ Department of Earth and Planetary Sciences, University of California, Santa Cruz, Santa Cruz, California \\ BRUCE T. ANDERSON \\ Department of Earth and Environment, Boston University, Boston, Massachusetts \\ SIMONA BORDONI \\ Environmental Science and Engineering, California Institute of Technology, Pasadena, California
}

(Manuscript received 28 September 2016, in final form 27 July 2017)

\begin{abstract}
Projections of amplified climate change in the Arctic are attributed to positive feedbacks associated with the retreat of sea ice and changes in the lapse rate of the polar atmosphere. Here, a set of idealized aquaplanet experiments are performed to understand the coupling between high-latitude feedbacks, polar amplification, and the large-scale atmospheric circulation. Results are compared to CMIP5. Simulated climate responses are characterized by a wide range of polar amplification (from none to nearly $15-\mathrm{K}$ warming, relative to the low latitudes) under $\mathrm{CO}_{2}$ quadrupling. Notably, the high-latitude lapse rate feedback varies in sign among the experiments. The aquaplanet simulation with the greatest polar amplification, representing a transition from perennial to ice-free conditions, exhibits a marked decrease in dry static energy flux by transient eddies. Partly compensating for the reduced poleward energy flux is a contraction of the Ferrel cell and an increase in latent energy flux. Enhanced eddy energy flux is consistent with the upper-tropospheric warming that occurs in all experiments and provides a remote influence on the polar lapse rate feedback. The main conclusions are that (i) given a large, localized change in meridional surface temperature gradient, the midlatitude circulation exhibits strong compensation between changes in dry and latent energy fluxes, and (ii) atmospheric eddies mediate the nonlinear interaction between surface albedo and lapse rate feedbacks, rendering the highlatitude lapse rate feedback less positive than it would be otherwise. Consequently, the variability of the circulation response, and particularly the partitioning of energy fluxes, offers insights into understanding the magnitude of polar amplification.
\end{abstract}

\section{Introduction}

Rapid warming of the Arctic is a robust result in projections of anthropogenic climate change, as well as an aspect of climate change already under way in the present climate. From an energy balance perspective, Arctic amplification of surface warming relative to lower latitudes may be caused by either local positive feedback mechanisms, convergence of heat from other latitudes, or reduction in heat taken up by the ocean,

Supplemental information related to this paper is available at the Journals Online website: https://doi.org/10.1175/ JCLI-D-16-0706.s1.

Corresponding author: Nicole Feldl,nfeldl@ucsc.edu whether by storage or ocean dynamics. Feedback analyses and ensemble modeling efforts (Hwang et al. 2011; Pithan and Mauritsen 2014) have revealed that increases in atmospheric heat transport make only minor contributions to warming averaged over the Arctic. Additionally, observed increases in North Atlantic heat uptake (Domingues et al. 2008) are consistent with projected changes in the Atlantic meridional overturning circulation that act to delay rather than enhance surface warming (Winton et al. 2014; Kostov et al. 2014). Hence, understanding atmospheric feedbacks is of primary importance for understanding the future warming of the Arctic. While the surface albedo feedbackassociated with an increase in absorbed shortwave radiation due to melting snow and sea ice-has long held an association with high-latitude climate change 
(Arrhenius 1896), recent studies have highlighted the additional importance of longwave temperature feedbacks, including the lapse rate feedback (Pithan and Mauritsen 2014; Winton 2006).

The surface albedo feedback and lapse rate feedback are both positive at high latitudes and dominate contributions to Arctic amplification; however, their meridional structures are quite different (Feldl and Roe 2013). The surface albedo feedback is strongly positive at high latitudes and zero elsewhere. The lapse rate feedback, on the other hand, represents various atmospheric processes that affect the lapse rate differently. Generally speaking, the lapse rate feedback is associated with deviations in tropospheric warming with altitude. In the tropics, the vertical structure of warming tends to follow moist adiabats and hence be enhanced aloft. As a result, outgoing longwave radiation is enhanced, which is a negative feedback relative to vertically uniform warming. However, at high latitudes, tropospheric warming instead tends to maximize near the surface. This bottom-heavy structure is typically found in the presence of boundary layer inversions, and it results in a relative decrease in planetary emission temperature (and hence reduced cooling to space)a positive feedback on surface temperature. Thus, the lapse rate feedback transitions from negative to positive from the equator to the poles.

Local Arctic processes, such as sea ice retreat and sea surface temperature (SST) changes (Deser et al. 2010; Serreze et al. 2009), influence the low-level atmospheric temperature. Additionally, the thermal structure aloft is set in part by large-scale turbulent eddies that act to stabilize the atmosphere. Eddies redistribute energy upward and poleward, with the magnitude of their flux scaling diffusively (Schneider and Walker 2006; Held 2007; Zurita-Gotor and Lindzen 2007; Jansen and Ferrari 2013). As a result, the meridional temperature gradient, which weakens under Arctic amplification, exerts a controlling influence on the extratropical lapse rate. O'Gorman (2011) expanded upon early studies based on dry theory to include the effect of latent heat release, which permits stability to increase in spite of the reduced meridional temperature gradient with warming. Recent work has emphasized the competition between local and nonlocal factors in influencing the lapse rate feedback and Arctic amplification in GCM simulations (Screen et al. 2012) and in analytic models (Payne et al. 2015; Cronin and Jansen 2016). Importantly, the different driving mechanisms have different fingerprints on the vertical structure of temperature change.

From the remote perspective, then, the lapse rate and its feedback are determined in part by the midlatitude circulation. Yet, feedbacks in turn modulate
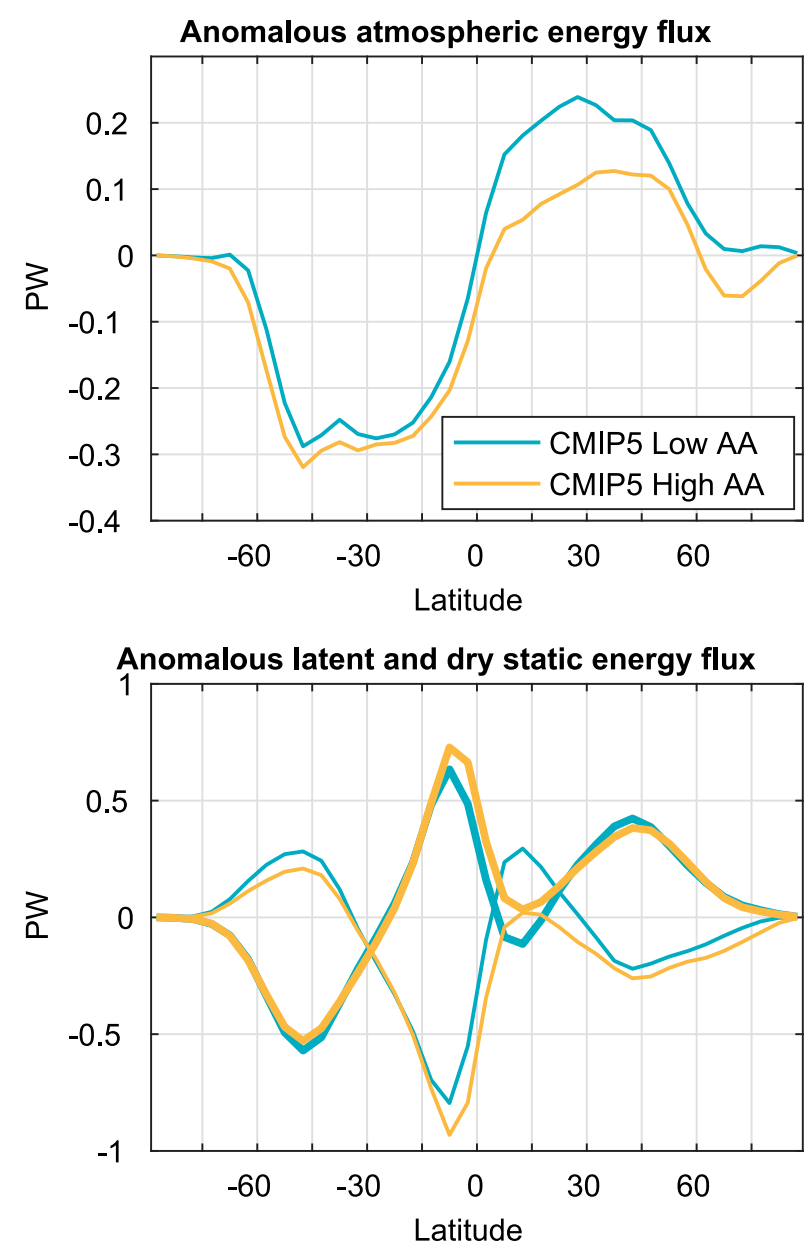

FIG. 1. Anomalous northward atmospheric energy flux for the CMIP5 RCP8.5 simulations with reduced Arctic amplification (low AA) and enhanced amplification (high AA) for the period 20062100. Models are composited based on an EOF analysis; see supplemental material for details. (bottom) Latent energy (thick lines) and dry static energy (thin lines) components of the total atmospheric energy flux. The decomposition methodology is the same as that discussed in section 2 .

atmospheric heat transport. Feedbacks are defined as additional radiative fluxes at the top of the atmosphere in response to an external climate forcing; in equilibrium, the forcing and the sum of the fluxes from feedbacks are together balanced by changes in atmosphere and ocean energy flux divergence (Zelinka and Hartmann 2012; Feldl and Roe 2013; Feldl and Bordoni 2016). In other words, in a slab-ocean aquaplanet model, any individual feedback acts to increase the atmospheric energy flux divergence at latitudes where the feedback is positive. A relationship between atmospheric heat transport and Arctic amplification is also evident in state-of-the-art coupled ocean-atmosphere models. Figure 1 shows the change in poleward energy flux based on 18 CMIP5 models forced by the RCP8.5 scenario. 
While more than two-thirds of the simulations exhibit anomalous divergence of atmospheric energy flux in the Arctic (not shown), the high Arctic amplification composite is additionally characterized by an anomalously equatorward energy flux north of $60^{\circ} \mathrm{N}$. This result suggests a limit on the increase in poleward energy flux, and motivates our investigation via a series of idealized experiments.

The importance of understanding changes in atmospheric heat transport is encapsulated in the related motivation: How do various aspects of the general circulation respond to enhanced greenhouse gas concentrations, and how does latent heat release modify the response? The impact of Arctic changes on midlatitude weather patterns, including jet stream position and variability, is a particular topic of debate. Though a few studies have linked weather extremes to high-latitude warming (Francis and Vavrus 2012), analyses of the observational record are hindered by large internal variability (Screen et al. 2014). A number of recent studies in fact suggest decreasing trends in cold weather extremes associated with Arctic warming (Schneider et al. 2015; Screen et al. 2015). Herein, projected changes in circulation are investigated in a simplified model framework and compared to more comprehensive coupled ocean-atmosphere models. By systematically manipulating the strength of the surface albedo feedback, we generate a wide range of Arctic-amplified climates, which mark the transition from perennial to seasonal to ice-free conditions. Our goals are to understand (i) how the compensation between dry and latent energy fluxes varies with the magnitude and structure of polar amplification and (ii) how the circulation mediates the nonlinear interaction between surface albedo and lapse rate feedbacks.

\section{Methods}

We employ the Geophysical Fluid Dynamics Laboratory Atmospheric Model (GFDL AM2.1; Delworth et al. 2006) in its aquaplanet configuration; the description of the experimental setup parallels that of Feldl et al. (2017). Model resolution is $2^{\circ}$ latitude by $2.5^{\circ}$ longitude. Orbital specifications include daily mean solar zenith angle, seasonally varying insolation, and zero eccentricity. The model is coupled to a $30-\mathrm{m}$ slab ocean with no oceanic heat transport. Sea ice formation is enabled by introducing an ocean albedo dependence on surface temperature; the surface albedo is increased where surface temperatures are less than $270 \mathrm{~K}$. Control and perturbation $\mathrm{CO}_{2}$ concentrations are 330 and $1320 \mathrm{ppm}$, respectively. The ice albedo $\alpha_{i}$ is systematically varied $(0.3,0.4,0.45,0.5)$ to manipulate the strength of the surface albedo feedback. The eight simulations are integrated for 40 years, with the exception of $\alpha_{i}=0.5$, which is run for 45 years (including 15 years of spinup), to serve as a run from which the others are branched. Monthly climatologies used in the following calculations are computed from 30 -yr periods.

Moist static energy flux divergence in the atmosphere is calculated from the difference between top-of-theatmosphere (TOA) net radiative flux $R_{T}$ and surface fluxes $F_{s}$ including radiative, latent heat, and sensible heat fluxes. The contribution of kinetic energy to the total atmospheric energy is small (Trenberth and Stepaniak 2003), and we omit it. Given that the storage of energy in the atmosphere is negligible in the annual average, the atmospheric energy flux (PW) is then simply the zonal and meridional integral of this quantity, that is,

$$
F_{a}=\int_{-\frac{\pi}{2}}^{\phi} \int_{0}^{2 \pi}\left(R_{T}-F_{s}\right) a^{2} \cos \phi d \lambda d \phi,
$$

where $a$ is the radius of Earth, $\phi$ is latitude, and $\lambda$ is longitude. The anomalous atmospheric energy flux is the difference between $4 \times \mathrm{CO}_{2}$ and $1 \times \mathrm{CO}_{2}$ simulations. Moist static energy $h$ may alternatively be computed as the sum of the dry static energy $C_{p} T+g z$ and latent energy $L_{v} q$, which gives us our first decomposition of the total atmospheric energy flux into latent and dry components, respectively:

$$
\begin{aligned}
F_{l} & =\int_{-\frac{\pi}{2}}^{\phi} \int_{0}^{2 \pi} L_{v}(E-P) a^{2} \cos \phi d \lambda d \phi, \text { and } \\
F_{d} & =\int_{-\frac{\pi}{2}}^{\phi} \int_{0}^{2 \pi}\left[R_{T}-F_{s}-L_{v}(E-P)\right] a^{2} \cos \phi d \lambda d \phi
\end{aligned}
$$

noting that in the annual mean, net evaporation (evaporation minus precipitation) $E-P$ is balanced by the vertical integral of the divergence of water vapor flux (Hwang and Frierson 2010; Trenberth and Stepaniak 2003).

A second decomposition of the atmospheric energy flux relies on the eddy and mean meridional circulations. Transient eddies are associated with deviations from the time mean, stationary eddies with deviations from the zonal mean, and the mean meridional circulation (MMC) with the zonal-mean circulations [Eq. (4.9) in Peixoto and Oort (1992)]. Specifically, the energy flux by the MMC is the vertical mass-weighted integral of the flux of zonal-mean monthly mean moist static energy by the zonal-mean monthly mean meridional wind:

$$
F_{m}=\frac{2 \pi a \cos \phi}{g} \int_{p_{s}}^{0}[v][h] d p,
$$


where here brackets $([\cdot])$ denote a zonal average. The use of monthly averages in this formalism means that the flux includes seasonal variability. The zonal-mean meridional wind is adjusted to be in mass balance following Hill et al. (2015). Stationary eddies do not form in these aquaplanet simulations, consistent with the absence of zonal asymmetries associated with continents, and as such they cannot transport momentum, heat, or moisture. Since we are restricted to monthly climatologies and since the stationary eddy flux is zero, ${ }^{1}$ the energy flux by transient eddies at synoptic time scales is best computed as the difference between the total atmospheric and MMC energy fluxes:

$$
F_{e}=F_{a}-F_{m}
$$

Finally, the latent and dry decomposition may also be performed on the MMC and eddy fluxes by considering only the latent energy contribution to moist static energy in Eq. (4) [see also Eq. (13.39) of Peixoto and Oort (1992)]:

$$
\begin{aligned}
F_{\mathrm{ml}} & =\frac{2 \pi a \cos \phi}{g} \int_{p_{s}}^{0} L_{v}[v][q] d p, \\
F_{\mathrm{md}} & =F_{m}-F_{\mathrm{ml}}, \\
F_{\mathrm{el}} & =F_{l}-F_{\mathrm{ml}}, \quad \text { and } \\
F_{\mathrm{ed}} & =F_{d}-F_{\mathrm{md}} .
\end{aligned}
$$

Subscripts $l, d, m$, and $e$ indicate latent, dry static, MMC, and eddy energy fluxes, respectively, and may be combined (e.g., $\mathrm{ml}$ is the latent energy flux by the MMC). In the next section, we perform a detailed decomposition of the anomalous heat (i.e., moist static energy) transport by the atmosphere for each albedo experiment, following the above equations.

\section{Results}

\section{a. Eddy energy flux}

The four aquaplanet experiments, in which the ice albedo value but not its areal extent is specified, exhibit a wide range of polar amplification under $4 \times$ $\mathrm{CO}_{2}$ (Fig. 2a). High-latitude surface temperature change is $5 \mathrm{~K}$ in the low-albedo experiment $\left(\alpha_{i}=0.3\right)$ and up to $24 \mathrm{~K}$ in the high-albedo experiment $\left(\alpha_{i}=0.5\right)$. The high-albedo experiment starts from the coldest climatology, with year-round sea ice cover to nearly $50^{\circ}$

\footnotetext{
${ }^{1}$ The stationary eddy energy flux is calculated as the vertical integral of $\left[v^{*} h^{*}\right]$ following Eq. (4), where (.*) denotes a deviation of the monthly mean from its zonal average.
}
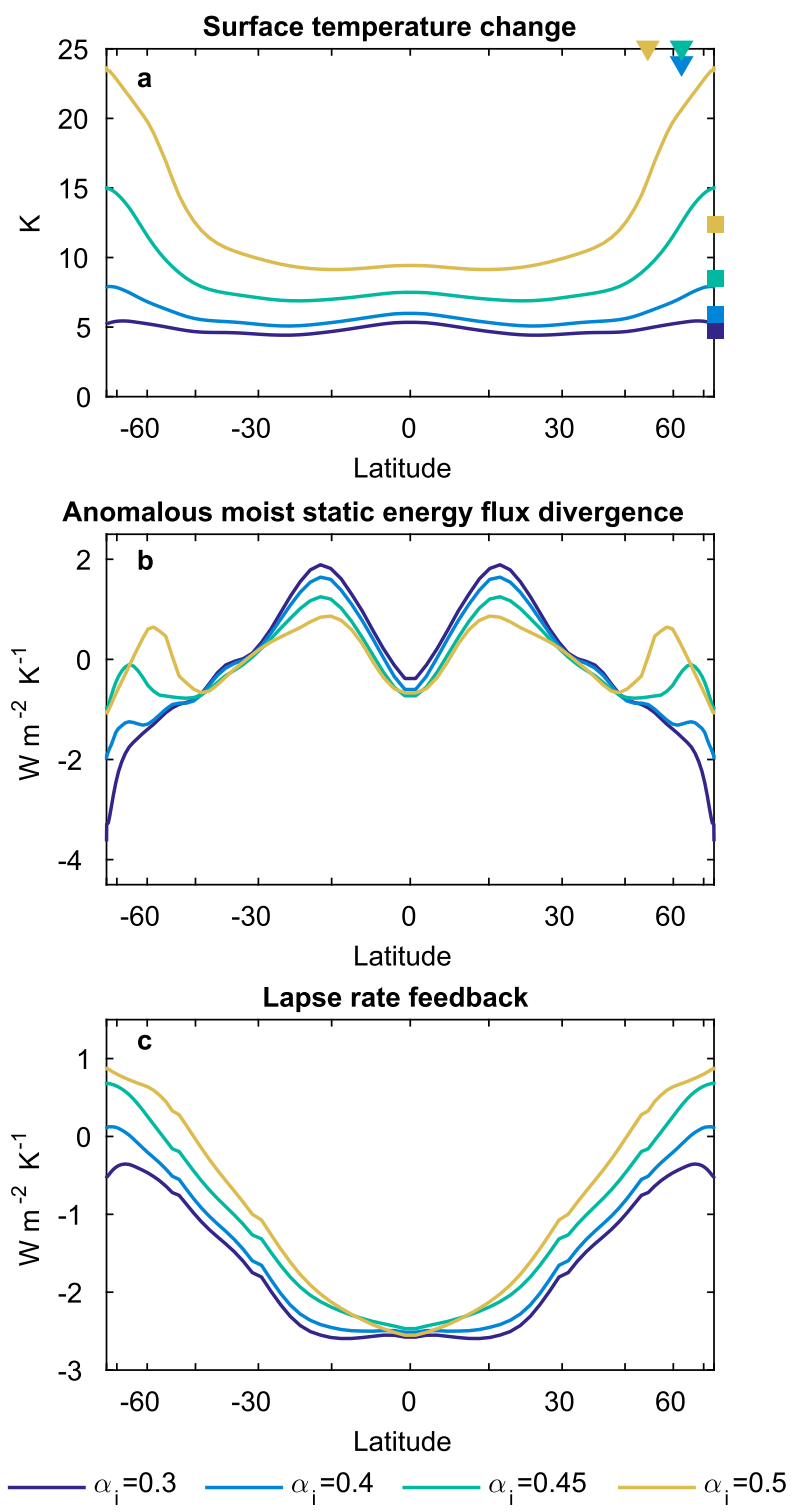

FIG. 2. (a) Annual-mean zonal-mean change in near-surface temperature $(\mathrm{K})$ under $4 \times \mathrm{CO}_{2}$ for the four aquaplanet experiments. Surface albedo values are 0.3 (purple), 0.4 (blue), 0.45 (green), and 0.5 (yellow). Global-mean warming is indicated on the $y$ axis. Latitude of the maximum change in meridional surface temperature gradient is indicated on the $x$ axis. (b) Zonal-mean anomalous moist static energy flux divergence $\left(\mathrm{W} \mathrm{m}^{-2} \mathrm{~K}^{-1}\right)$ calculated from the net radiative flux at the top of the atmosphere and normalized by the local surface air temperature change. (c) Zonalmean regional lapse rate feedback parameter.

latitude, and must warm substantially to reach the same end point as the low-albedo experiment, which only ever forms seasonal sea ice. Sea ice is absent in the equilibrium $4 \times \mathrm{CO}_{2}$ climate in all simulations, and the simulations all reside in the same warm climate state. Hence, differences in surface warming reflect differences in the initial mean state and climate feedbacks. 
Different climate feedbacks among the experiments result in different meridional structures of anomalous moist static energy flux divergence (Feldl et al. 2017). A strongly positive surface albedo feedback promotes anomalous divergence of energy flux from the region of sea ice retreat - in other words, a decrease in highlatitude energy flux convergence (Fig. 2b). This response is unique to the high-albedo experiment, which transitions from perennial ice cover to ice-free conditions. In contrast, the other experiments exhibit anomalous convergence of energy flux into the poles and divergence from the subtropics, strengthening the gradient of the climatological meridional energy flux divergence. As noted by Feldl et al. (2017) and illustrated in Fig. 2c, the lapse rate feedback, associated with deviations of tropospheric temperatures from vertically uniform warming, varies in sign among the experiments, which we return to discuss in section $3 \mathrm{~b}$. The absence of polar amplification in the low-albedo experiment is explained by the strong gradient in the net feedback from the poles to subtropics. Specifically, the high latitudes are characterized by a negative shortwave cloud feedback, a negative lapse rate feedback, and a negligible surface albedo feedback. This (lack of) warming pattern stands in stark contrast to previous aquaplanet experiments under perpetual equinox conditions (Roe et al. 2015).

In the low-albedo experiment, the increase in poleward energy flux peaks at 0.6 PW in the midlatitudes (Fig. 3a). Figure $3 b$ shows the decomposition into latent energy (thick lines) and dry static energy (thin lines) fluxes. Latent energy flux is equatorward in the tropical Hadley cell region and poleward in the extratropics. Under enhanced $\mathrm{CO}_{2}$, latent energy flux increases in all experiments, consistent with the moistening of the atmosphere with warming. In the low-albedo experiment, the extratropical latent energy flux is partly offset by a small decrease in dry static energy flux associated with very modest polar amplification of surface temperature. A key point to understanding the results is the proportionality between dry static energy flux and the meridional temperature gradient, consistent with the diffusive closures of this flux. Qualitatively, our results are consistent with Held and Soden (2006, see also their corrigendum) and Hwang et al. (2011), though our forcing, and hence responses, are larger. In particular, in the highalbedo experiment, polar amplification is much stronger and correspondingly, the decrease in dry static energy flux dominates, overcompensating for the increase in latent energy flux. As a result, the total atmospheric energy flux is zero at $50^{\circ}$ latitude in $\alpha_{i}=0.5$, with a region of dramatic anomalous energy flux divergence on the poleward flank of that minimum (i.e., $\sim 50^{\circ}-65^{\circ}$ ).

Alternately, we can decompose the total atmospheric heat transport into components associated with the MMC
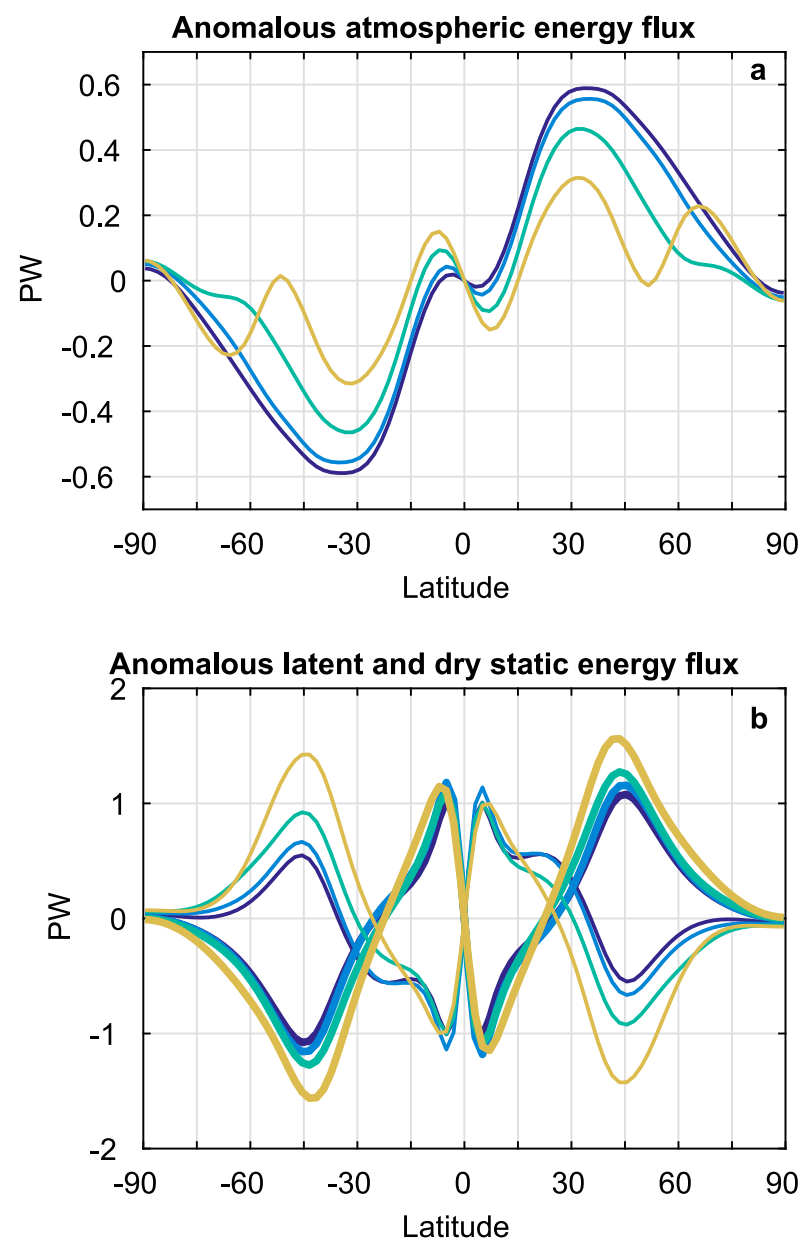

FIG. 3. (a) Anomalous northward atmospheric energy flux calculated as the moist static energy flux divergence integrated from the South Pole to the North Pole. Models are color coded as in the legend of Fig. 2. (b) Latent energy (thick lines) and dry static energy (thin lines) components of the total atmospheric flux.

and transient eddies (Fig. 4a). Climatologically, the extratropics are characterized by large poleward transient eddy energy flux and equatorward mean energy flux in the Ferrel cell. We may further subdivide the mean and eddy energy fluxes into their respective latent and dry components. Figure $4 \mathrm{~b}$ shows the total moist static energy flux quantified in terms of these four energy fluxes in the aquaplanet. The decomposition reveals that extratropical fluxes are dominated by dry static energy flux by transient eddies (thin dotted lines), particularly at high latitudes where the other fluxes are small. Partially offsetting the poleward flux are dry static energy fluxes by the mean meridional circulation (thin solid lines). The mean meridional circulation contributes only minimally to extratropical latent energy flux, and not at all poleward of $50^{\circ}$ latitude. We note that the greatest spread among energy fluxes occurs for the dry static energy flux by transient eddies in the midlatitudes. 

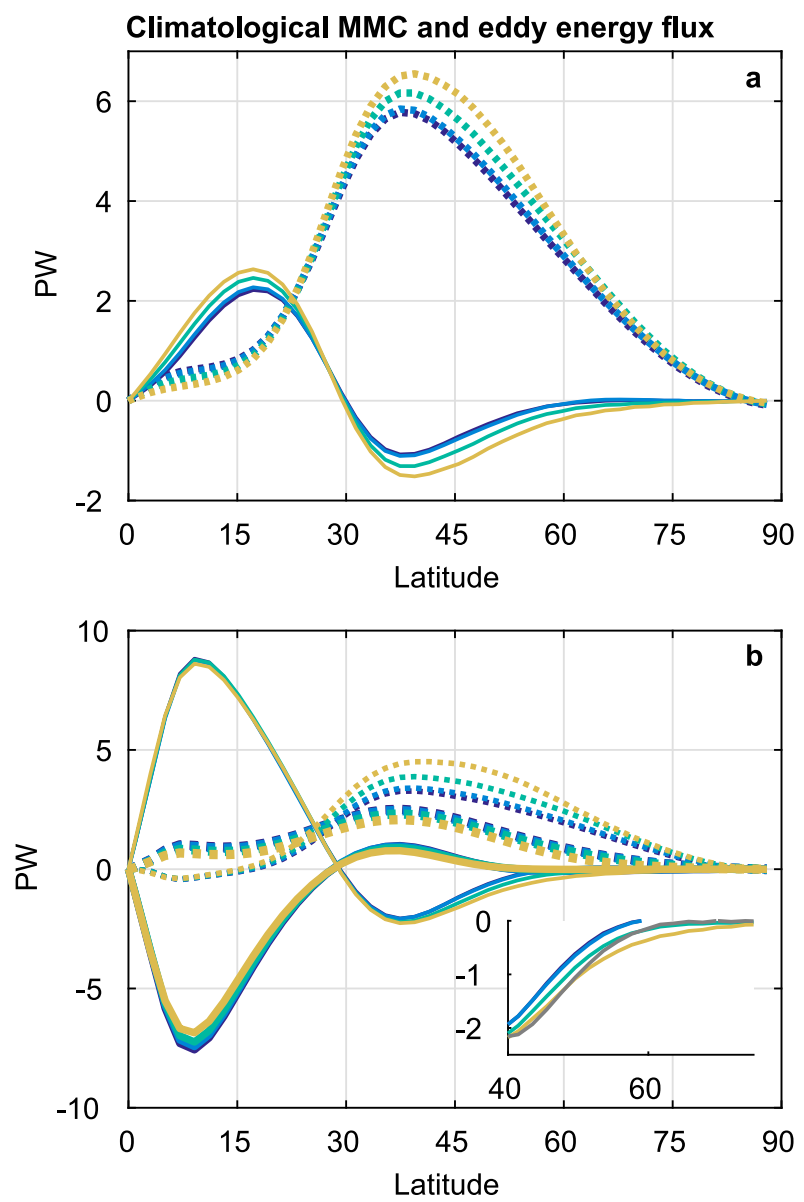

FIG. 4. (a) Climatological northward energy flux by the mean meridional circulation (solid lines) and by transient eddies (dotted lines). Note that stationary eddies are nonexistent in this zonally symmetric aquaplanet. Models are color coded as in the legend of Fig. 2. (b) Latent energy (thick lines) and dry static energy (thin lines) flux components of the MMC and eddy energy fluxes. The inset plot is an enlargement of the dry static energy flux by the $\mathrm{MMC}$, with the $4 \times \mathrm{CO}_{2}$ climatology in gray for reference.

To better understand the anomalous energy flux divergence exhibited by the high-albedo experiment, we turn to the anomalous mean meridional and eddy circulations (Fig. 5). The previously noted minimum in anomalous energy flux in $\alpha_{i}=0.5$ is due to the transient eddy energy flux - in particular a strong decrease in dry static energy flux that is only partly compensated for by an increase in latent energy flux. In contrast, in the other experiments the latent energy flux dominates and the high latitudes experience uninterrupted anomalous convergence of energy flux by the transient eddies. The contributions from the mean meridional circulation tend to be smaller, and differ in sign among experiments in the midlatitudes. This differential response is evidence of a shift in the position of the Ferrel cell. Climatologically, the poleward extent of the Ferrel cell ranges
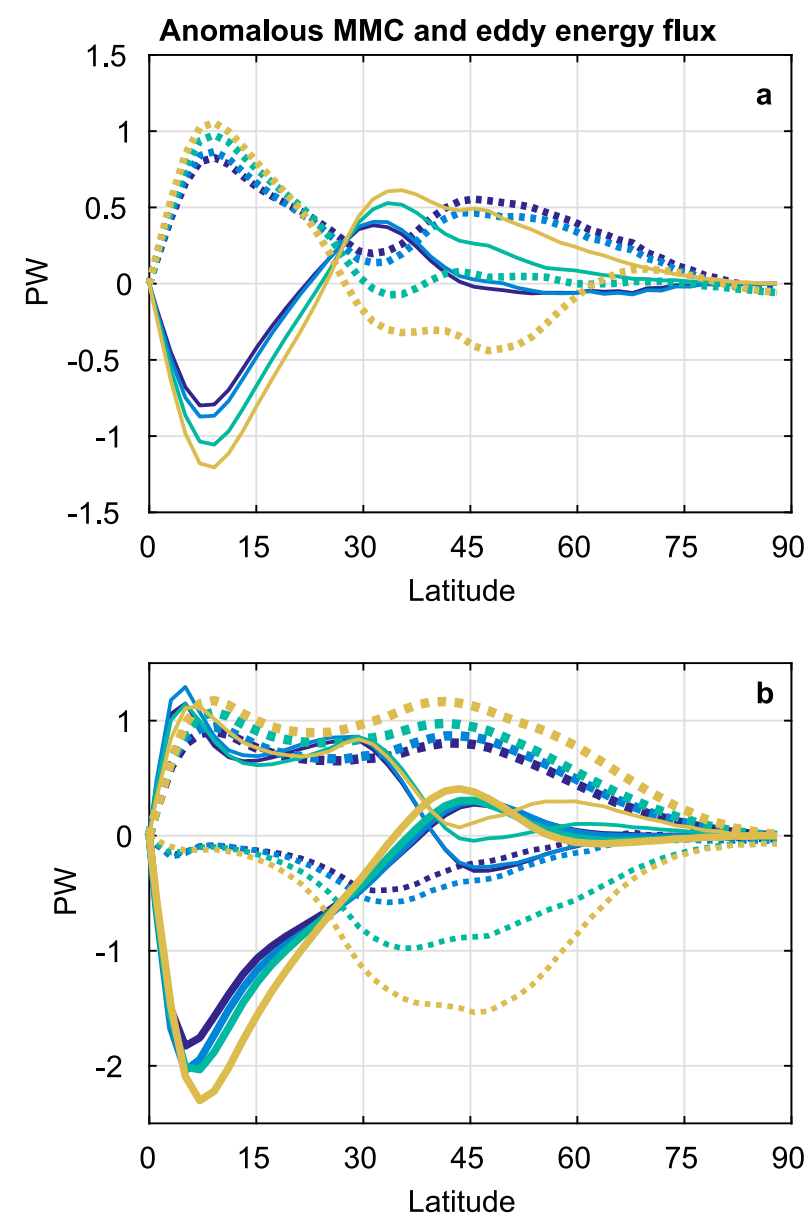

FIG. 5. (a) Anomalous northward energy flux by the mean meridional circulation (solid lines) and by transient eddies (dotted lines). Models are color coded as in the legend of Fig. 2. (b) Latent energy (thick lines) and dry static energy (thin lines) components of the MMC and eddy energy fluxes.

between $54^{\circ}\left(\alpha_{i}=0.3\right)$ and $63^{\circ}\left(\alpha_{i}=0.5\right)$ latitude for the aquaplanet simulations; under warming, the edge migrates to $60^{\circ}$ in all experiments. Hence, a poleward expansion of the Ferrel cell manifests as an anomalous equatorward energy flux by the MMC, and a narrowing of the Ferrel cell as an anomalous poleward energy flux (cf. Fig. 4 inset).

The strong compensation in the high-albedo experiment is influenced by both the magnitude of the reduction in meridional surface temperature gradient and the localization of that change approximately $12^{\circ}$ farther equatorward than in the other experiments (see $x$ axis of Fig. 2a). The magnitude of the gradient change is consistent with the large decrease in dry static energy flux by transient eddies, and hence in the total dry static energy flux. However, subtle differences in the shape of the anomalous energy fluxes, characterized by a poleward shift in the peak for the $\alpha_{i}=0.5$ experiment, are 


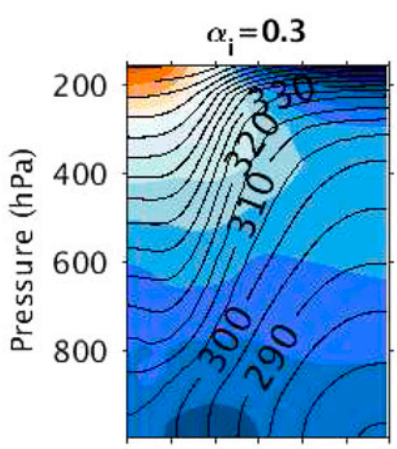

0153045607590 Latitude

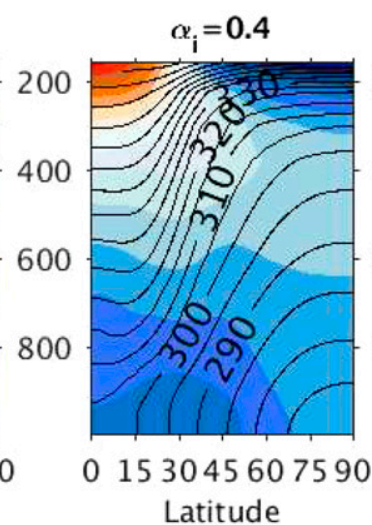

Latitude

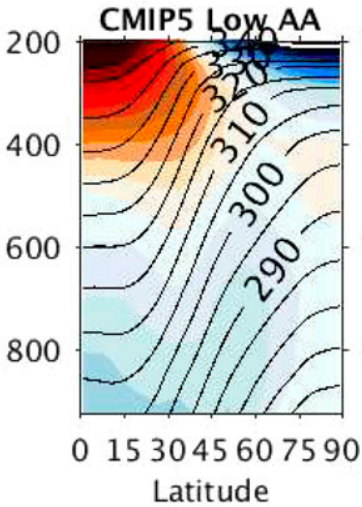

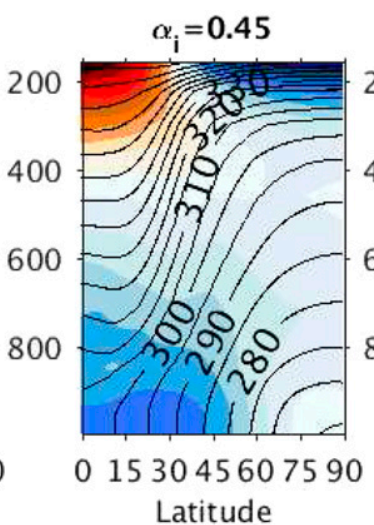

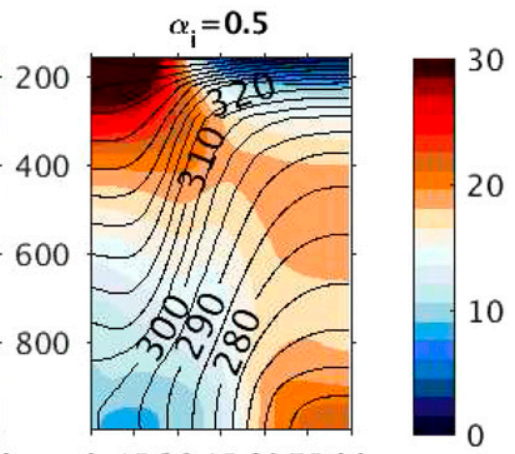

0153045607590

Latitude

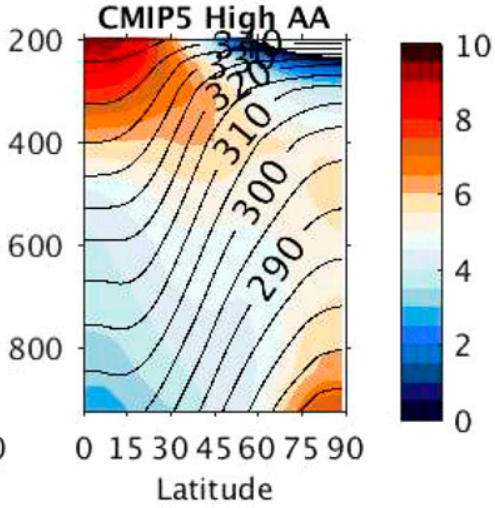

FIG. 6. (top) Climatological potential temperature (contours; 5-K interval) and change under $4 \times \mathrm{CO}_{2}$ for each of the four aquaplanet experiments. (bottom) The multimodel mean potential temperature for the CMIP5 RCP8.5 simulations with reduced Arctic amplification (low AA) and enhanced amplification (high AA) for the period 2006-2100. Models are composited based on an EOF analysis; see supplemental material for details. Note the CMIP5 color scale covers one-third the range of that of the aquaplanet.

explained by the influence of the sea ice edge on the latitude of the maximum gradient change. Poleward of that latitude, the anomalous dry static energy flux by transient eddies falls off rapidly. As a result, the increase in eddy latent energy flux dominates in the Arctic (Fig. 5a), resulting in a double hump in anomalous atmospheric energy flux (Fig. 3a). In contrast, the latitude of maximum surface temperature gradient change does not vary between the $\alpha_{i}=0.4$ and $\alpha_{i}=0.45$ experiments, and differences may only be attributed to the magnitude and not structure of the polar amplification.

\section{b. Polar lapse rate feedback}

Feedbacks drive changes in atmospheric heat transport, though they are themselves modulated by the climatological heat transport. We have demonstrated that, in the high-albedo experiment, the anomalous divergence of atmospheric energy flux from the region of positive surface albedo and lapse rate feedbacks is accomplished by a decrease in dry static energy flux by transient eddies. Partially offsetting that tendency is the contraction of the Ferrel cell and the increase in latent energy flux, which both increase poleward energy flux. We argue that the eddy energy flux is critical for the vertical structure of polar warming and the magnitude of the lapse rate feedback.

Figure 6 shows the vertical structure of potential temperature change in the four experiments. Two regions of warming are found at high latitudes: nearsurface warming confined to the boundary layer and warming aloft in the upper troposphere $(400-600 \mathrm{hPa}$ ); both are particularly apparent in $\alpha_{i}=0.5$. Contours show the control climatology. Relative to no change in lapse rate (i.e., uniform temperature change with height), bottom-heavy warming decreases the emission temperature, a positive feedback on surface temperature. The location of this warming structure supports the influence of the surface albedo modification on the lapse rate feedback. In particular, extensive sea ice retreat in the high-albedo experiments leads to an increase in absorbed shortwave radiation. The high-albedo experiments also exhibit boundary layer inversions in the initial mean state (not shown), which the strong bottomheavy warming erodes. Given this vertical structure of 
warming, it is unsurprising that the polar lapse rate feedback is positive in the high-albedo experiments and negative in the low-albedo experiments (Fig. 2c). The polar amplification of surface warming-and corresponding weakening of the meridional temperature gradient-is also consistent with the noted decrease in dry static energy flux by transient eddies.

The mechanism controlling upper-tropospheric temperature is more ambiguous. Previous work has suggested the role of atmospheric heat transport in warming the high-latitude upper troposphere (e.g., Alexeev et al. 2005; Graversen et al. 2008). Since enhanced warming aloft occurs in all four experiments (unlike bottomheavy warming), a straightforward explanation is that any remote contribution to warming would be associated with an anomalous convergence of energy flux that likewise persists in all experiments and influences high latitudes: energy flux by transient eddies (Fig. 5a). Though interpretation is complicated by our focus on vertically integrated fluxes, future work will seek to identify the sources of this warming structure that reduces the magnitude of the (typically positive) highlatitude lapse rate feedback.

\section{c. Precipitation}

A secondary effect of changes in latent energy flux is the increase in precipitation at latitudes of anomalous convergence of moisture flux. To evaluate whether the aquaplanet simulations show the anticipated pattern of precipitation changes, we perform a decomposition of the water vapor budget. The change in annual-mean net precipitation is balanced by the change in vertically integrated water vapor flux convergence:

$$
\Delta[P-E]=-\Delta \int_{p_{s}}^{0} \nabla \cdot[v q] \frac{d p}{g} .
$$

The precipitation response is described by an enhancement of the climatological net precipitation, consistent with Held and Soden (2006). Net precipitation changes in the subtropics are dominated by enhanced evaporation (not shown). We note that since our sea ice formulation is an ocean-albedo dependence on temperature, evaporation may be less inhibited relative to realistic representations. In the extratropics, the highalbedo experiment exhibits the largest increases in net precipitation, as well as an equatorward shift in the storm-track precipitation (Fig. 7a). The change in net precipitation may further be characterized, following Merlis et al. (2013), in terms of components associated with dynamical changes $(q \Delta v)$, thermodynamical changes $\left(v H \Delta q_{s}\right)$, relative humidity changes $\left(v q_{s} \Delta H\right)$, transient eddies (calculated as a residual), as well as the covariance between $\Delta v$ and $\Delta q$ (quadratic component); $q_{s}$ is the saturation specific humidity and $H$ is the relative humidity. The calculation is performed on monthly mean fields so that the dynamic component, for instance, refers to dynamical changes in the mean moisture flux convergence.

The dominance of any given term depends on the latitude of interest. In polar regions, the net precipitation mirrors the moisture flux convergence associated with submonthly transients (Fig. 7b). A particularly large excursion is evident in the high-albedo experiment, consistent with the enhanced poleward latent energy flux by transient eddies. However, in the midlatitudes, the mean thermodynamic and, to a lesser degree, dynamic components make larger contributions to the spread among experiments. In particular, warming leads to an increase in net precipitation that peaks at $45^{\circ}$ (Fig. 7c) and a distinct poleward shift of the storm track in the low-albedo experiments (note the dipole in Fig. $7 \mathrm{~d}$ ). In the high-albedo experiments, though, the dynamical signature on precipitation is limited to that of a weakening Ferrel cell. Hence, the Ferrel cell expands poleward and the dynamical precipitation consequently increases at high latitudes, but only so long as the polar amplification is weak.

\section{Summary and discussion}

Arctic amplification in projections of anthropogenic climate change is dominated by positive feedbacks associated with the retreat of sea ice and changes in the thermal structure of the polar atmosphere. We perform a series of aquaplanet experiments to investigate a range of amplifications within an idealized modeling framework. The simulation with the greatest Arctic amplification (24-K local warming), representing a transition from perennial to ice-free conditions, exhibits a marked decrease in atmospheric energy flux convergence at the sea ice margin. The anomalous divergence of energy flux is accomplished by a decrease in dry static energy flux by the transient eddies. Partly compensating the reduced poleward energy flux is a contraction of the Ferrel cell and an increase in latent energy flux. In simulations with less Arctic amplification and weaker local feedbacks, the increase in latent energy flux instead dominates; in addition, the Ferrel cell expands poleward. Enhanced energy flux by transient eddies is consistent with the upper-tropospheric Arctic warming that occurs in all experiments irrespective of the sign of the lapse rate feedback. This eddy response modulates the lapse rate feedback, rendering it less positive than it would be in the absence of enhanced eddy energy flux aloft. Last, it is only for a specific parameter regime, characterized by 

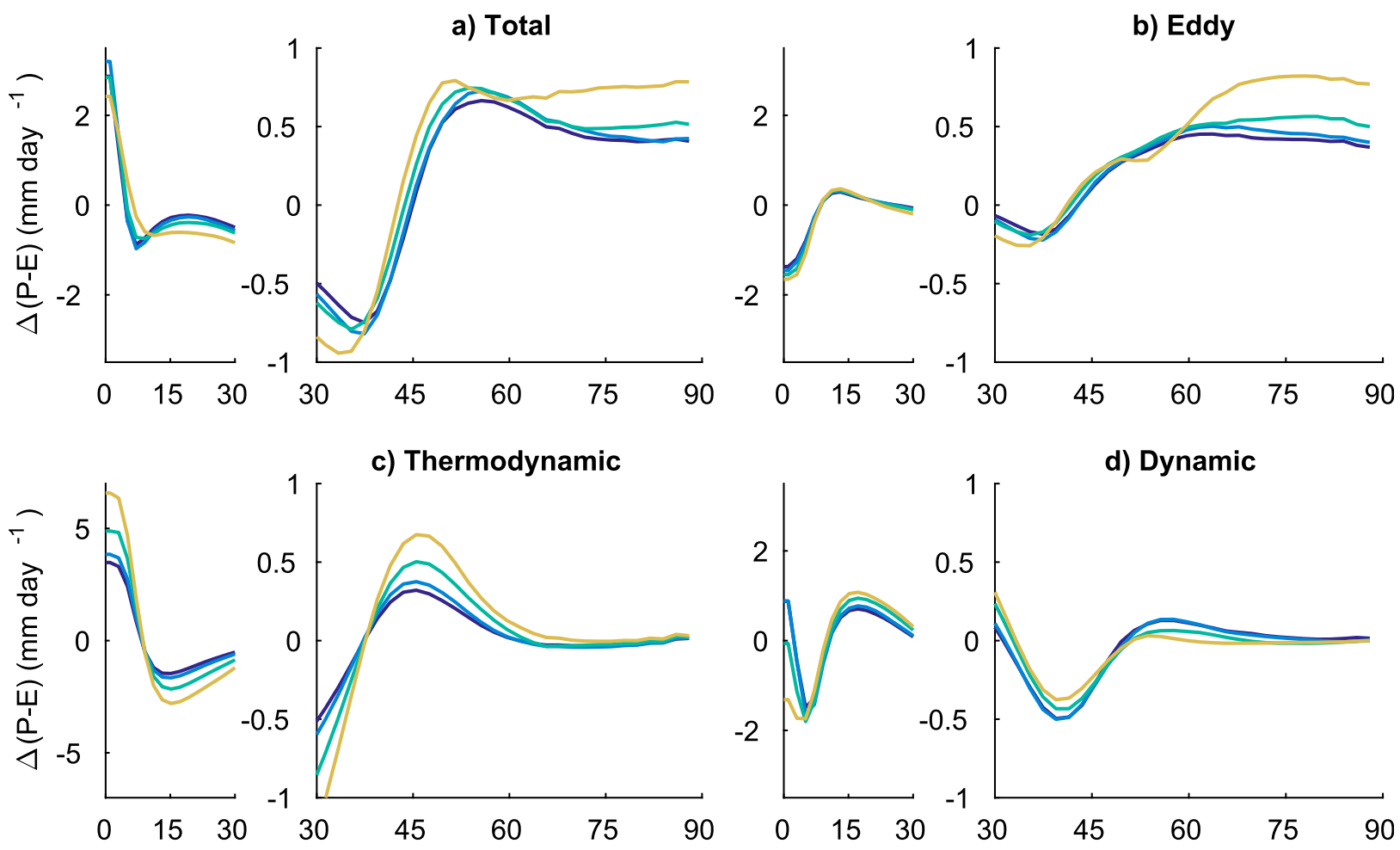

d) Dynamic

e) Quadratic
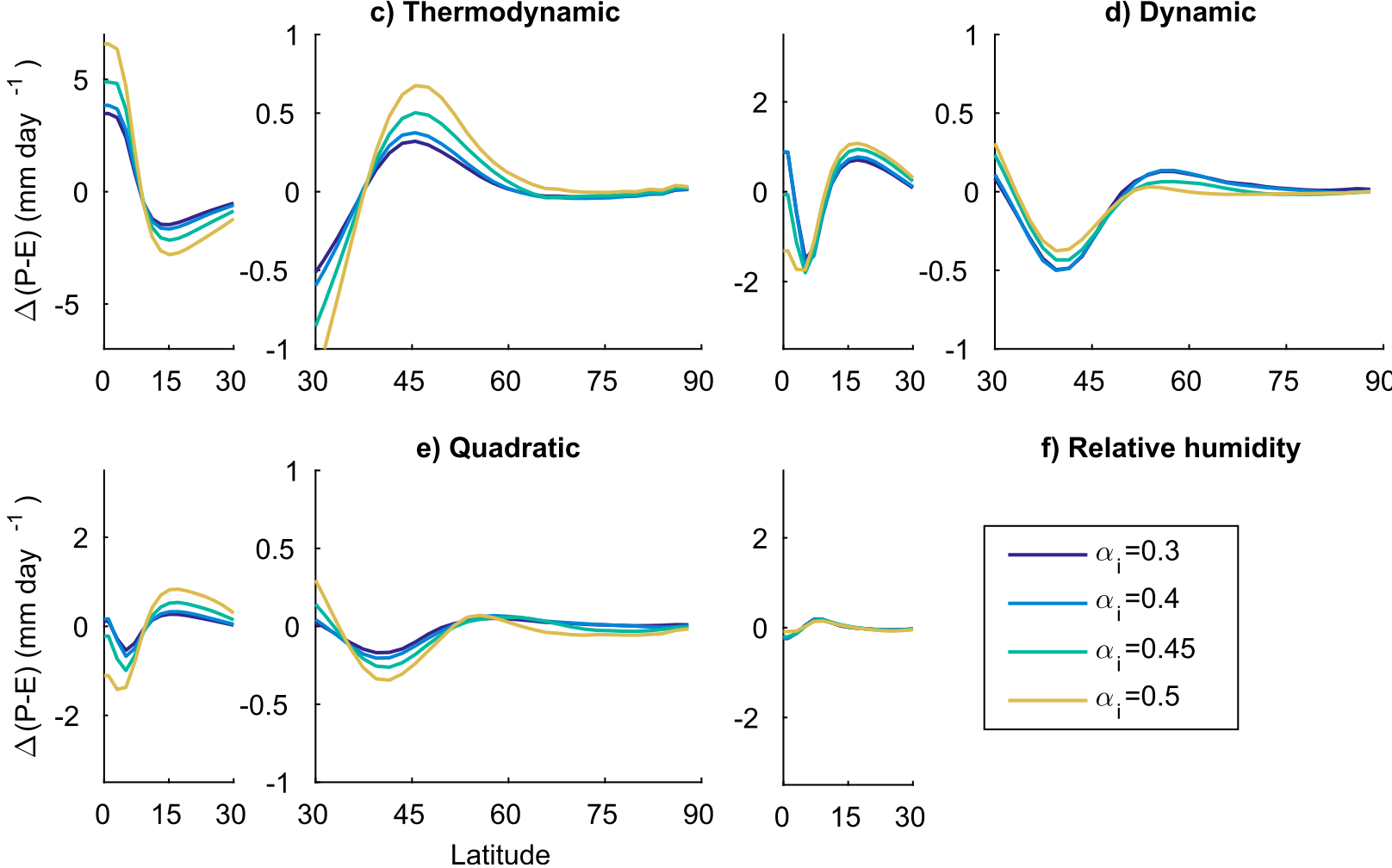

f) Relative humidity

FIG. 7. Annual-mean change in (a) net precipitation $\left(P-E, \mathrm{~mm} \mathrm{day}^{-1}\right)$ decomposed into (b) eddy, (c) thermodynamic, (d) dynamic, and (e) quadratic contributions. For reference, the tropical precipitation response is shown to the left of each panel. Changes in water vapor flux convergence associated with relative humidity changes are negligible in the extratropics.

midlatitude sea ice in the control climate and hence a more equatorward expression of the reduction in meridional surface temperature gradient, that the circulation exhibits perfect compensation between changes in latent and dry static energy fluxes, and only for a narrow latitude range (Fig. 8).

Energy transport by transient atmospheric eddies lies at the core of our results. Polar amplification, by reducing the meridional temperature gradient, results in a decrease in dry static energy flux. Atmospheric moistening, by way of the Clausius-Clapeyron relation, results in an increase in latent energy flux. Enhanced extratropical precipitation, associated with the convergence of that moisture flux on eddy time scales, is greatest for the experiment with the greatest degree of polar amplification. However, under strong warming, the relative importance of latent energy flux to the total energy transport is reduced. While our discussion has focused on large-scale fluxes of latent energy, a role for extratropical moist convection is not precluded in warmer climates (Emanuel 1988; Juckes 2000). Two caveats bear repeating: 1) Transient eddies are calculated as the difference between the total atmospheric energy flux and the energy flux by the mean meridional 


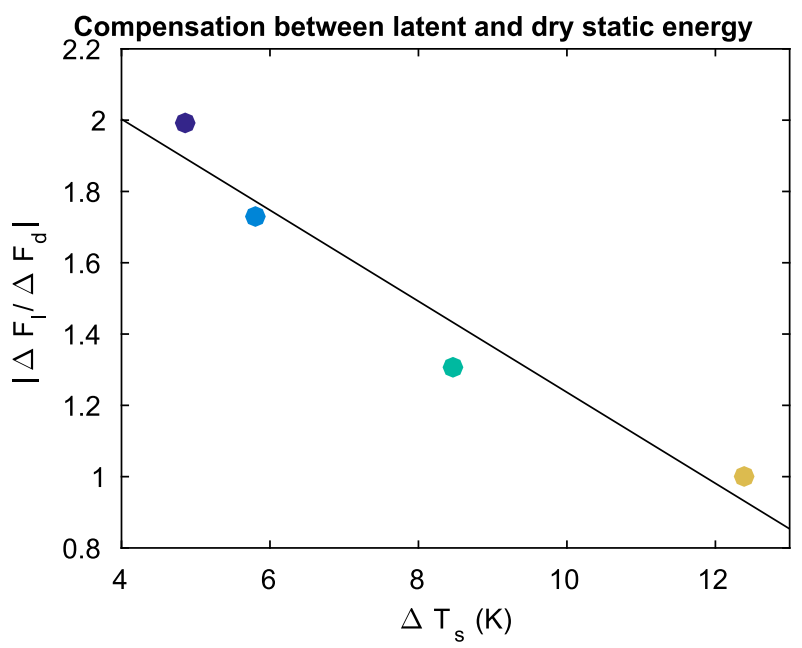

FIG. 8. Ratio of anomalous latent energy flux to dry static energy flux magnitudes (Fig. 3) evaluated at $50^{\circ}$ latitude. The linear fit indicates a decrease of $12.8 \%$ per degree of global-mean surface temperature change. Models are color coded as in the legend of Fig. 2.

circulation. We did not have access to the submonthly data required for an explicit calculation. 2) Stationary eddies, which may also contribute to Arctic warming (e.g., Baggett et al. 2016) do not form in these simulations, consistent with the absence of zonal asymmetries. While this represents a departure of the aquaplanet from more realistic configurations, the benefit is a straightforward definition of eddies and their effect on anomalous energy flux convergence at high latitudes.

In this study, we have sought to contextualize the idealized simulations by presenting key results from a more comprehensive suite of simulations forced by the RCP8.5 scenario of CMIP5. In both CMIP5 as well as in our aquaplanet experiments, anomalous divergence (i.e., reduced convergence) of atmospheric energy flux occurs in polar regions for simulations that exhibit strong polar amplification. In CMIP5, that divergence is characterized by a decrease in poleward energy flux. In the aquaplanet, we find reduced convergence in a single experiment $\left(\alpha_{i}=0.5\right)$ and at lower latitudes, controlled by the extensive sea ice in the $1 \times \mathrm{CO}_{2}$ climate. Since the sea ice margin is a focus for the decrease in meridional temperature gradient, the farther equatorward the ice extends into regions of higher eddy activity, the greater the potential for eddy energy flux to be affected. We note that small-scale variations in the meridional temperature gradient as a function of altitude may also lead to complex influences on the structure of eddy energy flux, especially in the experiments not dominated by vertically extensive polar warming. Additionally, boundary layer warming is present but damped in the CMIP5 projections, relative to that of the high-albedo aquaplanet experiment (Fig. 6). The reduced response may be due to the persistence of wintertime sea ice or to an increase in ocean heat uptake, either of which can affect the thermal structure of the polar atmosphere. The high-albedo aquaplanet experiment, on the other hand, is ice free and inversion free in its $4 \times \mathrm{CO}_{2}$ climate and has no ocean dynamics; moreover, the simplified sea ice representation is solely a modification of ocean albedo and not of other surface fluxes. Since bottom-heavy warming is a characteristic feature of a positive lapse rate feedback, one might anticipate the Arctic lapse rate feedback in the $\alpha_{i}=$ 0.5 experiment to be relatively large. Indeed, the value is $0.9 \mathrm{~W} \mathrm{~m}^{-2} \mathrm{~K}^{-1}$ (for reference, the ensemble-mean Arctic lapse rate feedback in the CMIP5 abrupt $4 \times \mathrm{CO}_{2}$ experiments peaks at $0.72 \mathrm{~W} \mathrm{~m}^{-2} \mathrm{~K}^{-1}$; Feldl and Bordoni 2016). The high-albedo aquaplanet exaggerates the sea ice response relative to CMIP5 simulations in order to elucidate the effect of polar amplification on atmospheric circulation.

Previous work has demonstrated that surface albedo and lapse rate feedbacks interact considerably at high latitudes. For instance, Graversen et al. (2014) show that when the surface albedo feedback is suppressed, the surface amplification of warming is smaller, as expected, but in addition, the lapse rate feedback is less positive. Similarly, a positive lapse rate feedback induces highlatitude surface warming, which further melts sea ice and enhances the surface albedo feedback. Cronin and Jansen (2016) propose an additional pathway for remote influences on the lapse rate feedback. They demonstrate a negative lapse rate feedback when advective heat flux convergence wins out over destabilization by surface shortwave absorption. In the experiments herein, we likewise find that when polar amplification is moderate, energy flux by transient eddies is consistent with uppertropospheric warming that contributes to a neutral or even negative lapse rate feedback (Fig. 2c). When polar amplification is strong, warming is bottom heavy and the lapse rate feedback is positive, further enhancing polar amplification of surface temperatures. In this case, the effect of enhanced atmospheric heat transport is to provide a mediating effect on the feedback. The high-latitude lapse rate feedback is less positive than it would be in the absence of an eddy response.

Given atmospheric moistening, the poleward transport of (latent) energy will be favored up to the point at which eddy velocities and/or mixing lengths decrease. It is important to note that, rather than suppressing the surface albedo feedback as in previous studies (Graversen et al. 2014; Hall 2004; Mauritsen et al. 2013), we modify its magnitude, and hence the sensitivity of sea 
ice to warming. Among the resulting wide range of simulated climates, our low-albedo experiment, which barely forms wintertime sea ice in the $1 \times \mathrm{CO}_{2}$ climate, may provide insights into equable paleoclimates. In the absence of sea ice, enhanced atmospheric energy flux is effective at warming high latitudes - though not to the extent of amplifying that warming relative to low latitudes. On the other hand, a climate system with icy high latitudes hosts a competition between local and remote controls on the vertical structure of Arctic warming. The outcome of this tug-of-war determines the strength of the lapse rate feedback and hence Arctic amplification.

Acknowledgments. We thank two anonymous reviewers for their helpful comments on the manuscript and the editor, Peter Huybers. This work also benefited from discussions with Tim Merlis. NF was supported by the National Science Foundation (AGS-1524569).

\section{REFERENCES}

Alexeev, V. A., P. L. Langen, and J. R. Bates, 2005: Polar amplification of surface warming on an aquaplanet in "ghost forcing" experiments without sea ice feedbacks. Climate Dyn., 24, 655-666, doi:10.1007/s00382-005-0018-3.

Arrhenius, S., 1896: XXXI: On the influence of carbonic acid in the air upon the temperature of the ground. London, Edinburgh, Dublin Philos. Mag. J. Sci., 41 (251), 237-276.

Baggett, C., S. Lee, and S. Feldstein, 2016: An investigation of the presence of atmospheric rivers over the North Pacific during planetary-scale wave life cycles and their role in Arctic warming. J. Atmos. Sci., 73, 4329-4347, doi:10.1175/ JAS-D-16-0033.1.

Cronin, T. W., and M. F. Jansen, 2016: Analytic radiative-advective equilibrium as a model for high-latitude climate. Geophys. Res. Lett., 43, 449-457, doi:10.1002/2015GL067172.

Delworth, T. L., and Coauthors, 2006: GFDL's CM2 global coupled climate models. Part I: Formulation and simulation characteristics. J. Climate, 19, 643-674, doi:10.1175/JCLI3629.1.

Deser, C., R. Tomas, M. Alexander, and D. Lawrence, 2010: The seasonal atmospheric response to projected Arctic sea ice loss in the late twenty-first century. J. Climate, 23, 333-351, doi:10.1175/2009JCLI3053.1.

Domingues, C. M., J. A. Church, N. J. White, P. J. Gleckler, S. E. Wijffels, P. M. Barker, and J. R. Dunn, 2008: Improved estimates of upper-ocean warming and multi-decadal sea-level rise. Nature, 453, 1090-1093, doi:10.1038/nature07080.

Emanuel, K. A., 1988: Observational evidence of slantwise convective adjustment. Mon. Wea. Rev., 116, 1805-1816, doi:10.1175/1520-0493(1988)116<1805:OEOSCA > 2.0.CO;2.

Feldl, N., and G. H. Roe, 2013: The nonlinear and nonlocal nature of climate feedbacks. J. Climate, 26, 8289-8304, doi:10.1175/ JCLI-D-12-00631.1.

— response through regional climate feedbacks. J. Climate, 29, 613-622, doi:10.1175/JCLI-D-15-0424.1.

,-- , and T. M. Merlis, 2017: Coupled high-latitude climate feedbacks and their impact on atmospheric heat transport. J. Climate, 30, 189-201, doi:10.1175/JCLI-D-16-0324.1.
Francis, J. A., and S. J. Vavrus, 2012: Evidence linking Arctic amplification to extreme weather in mid-latitudes. Geophys. Res. Lett., 39, L06801, doi:10.1029/2012GL051000.

Graversen, R. G., T. Mauritsen, M. Tjernstrom, E. Kallen, and G. Svensson, 2008: Vertical structure of recent Arctic warming. Nature, 451, 53-56, doi:10.1038/nature06502.

—, P. L. Langen, and T. Mauritsen, 2014: Polar amplification in CCSM4: Contributions from the lapse rate and surface albedo feedbacks. J. Climate, 27, 4433-4450, doi:10.1175/ JCLI-D-13-00551.1.

Hall, A., 2004: The role of surface albedo feedback in climate. J. Climate, 17, 1550-1568, doi:10.1175/1520-0442(2004)017<1550: TROSAF $>2.0 . \mathrm{CO} ; 2$.

Held, I. M., 2007: Progress and problems in large-scale atmospheric dynamics. The Global Circulation of the Atmosphere, T. Schneider and A. H. Sobel, Eds., Princeton University Press, 1-21.

- and B. J. Soden, 2006: Robust responses of the hydrological cycle to global warming. J. Climate, 19, 5686-5699, doi:10.1175/JCLI3990.1.

Hill, S. A., Y. Ming, and I. M. Held, 2015: Mechanisms of forced tropical meridional energy flux change. J. Climate, 28, 17251742, doi:10.1175/JCLI-D-14-00165.1.

Hwang, Y.-T., and D. M. W. Frierson, 2010: Increasing atmospheric poleward energy transport with global warming. Geophys. Res. Lett., 37, L24807, doi:10.1029/2010GL045440.

,$- \ldots$, and J. E. Kay, 2011: Coupling between Arctic feedbacks and changes in poleward energy transport. Geophys. Res. Lett., 38, L17704, doi:10.1029/2011GL048546.

Jansen, M., and R. Ferrari, 2013: Equilibration of an atmosphere by adiabatic eddy fluxes. J. Atmos. Sci., 70, 2948-2962, doi:10.1175/JAS-D-13-013.1.

Juckes, M. N., 2000: The static stability of the midlatitude troposphere: The relevance of moisture. J. Atmos. Sci., 57, 3050-3057, doi:10.1175/1520-0469(2000)057<3050:TSSOTM >2.0.CO;2.

Kostov, Y., K. C. Armour, and J. Marshall, 2014: Impact of the Atlantic meridional overturning circulation on ocean heat storage and transient climate change. Geophys. Res. Lett., $\mathbf{4 1}$, 2108-2116, doi:10.1002/2013GL058998.

Mauritsen, T., R. G. Graversen, D. Klocke, P. L. Langen, B. Stevens, and L. Tomassini, 2013: Climate feedback efficiency and synergy. Climate Dyn., 41, 2539-2554, doi:10.1007/ s00382-013-1808-7.

Merlis, T. M., T. Schneider, S. Bordoni, and I. Eisenman, 2013: The tropical precipitation response to orbital precession. J. Climate, 26, 2010-2021, doi:10.1175/JCLI-D-12-00186.1.

O'Gorman, P. A., 2011: The effective static stability experienced by eddies in a moist atmosphere. J. Atmos. Sci., 68, 75-90, doi:10.1175/2010JAS3537.1.

Payne, A. E., M. F. Jansen, and T. W. Cronin, 2015: Conceptual model analysis of the influence of temperature feedbacks on polar amplification. Geophys. Res. Lett., 42, 9561-9570, doi:10.1002/2015GL065889.

Peixoto, J. P., and A. H. Oort, 1992: Physics of Climate. SpringerVerlag, 520 pp.

Pithan, F., and T. Mauritsen, 2014: Arctic amplification dominated by temperature feedbacks in contemporary climate models. Nat. Geosci., 7, 181-184, doi:10.1038/ngeo2071.

Roe, G. H., N. Feldl, K. C. Armour, Y.-T. Hwang, and D. M. W. Frierson, 2015: The remote impacts of climate feedbacks on regional climate predictability. Nat. Geosci., 8, 135-139, doi:10.1038/ngeo2346.

Schneider, T., and C. C. Walker, 2006: Self-organization of atmospheric macroturbulence into critical states of weak nonlinear 
eddy-eddy interactions. J. Atmos. Sci., 63, 1569-1586, doi:10.1175/JAS3699.1.

, T. Bischoff, and H. Płotka, 2015: Physics of changes in synoptic midlatitude temperature variability. J. Climate, $\mathbf{2 8}$, 2312-2331, doi:10.1175/JCLI-D-14-00632.1.

Screen, J. A., C. Deser, and I. Simmonds, 2012: Local and remote controls on observed Arctic warming. Geophys. Res. Lett., 39, L10709, doi:10.1029/2012GL051598.

,,--- - - and R. Tomas, 2014: Atmospheric impacts of Arctic sea-ice loss, 1979-2009: Separating forced change from atmospheric internal variability. Climate Dyn., 43, 333-344, doi:10.1007/s00382-013-1830-9.

- _ - - , and L. Sun, 2015: Reduced risk of North American cold extremes due to continued Arctic sea ice loss. Bull. Amer. Meteor. Soc., 96, 1489-1503, doi:10.1175/ BAMS-D-14-00185.1.

Serreze, M. C., A. P. Barrett, J. C. Stroeve, D. N. Kindig, and M. M. Holland, 2009: The emergence of surface-based Arctic amplification. Cryosphere, 3, 11-19, doi:10.5194/tc-3-11-2009.
Trenberth, K. E., and D. P. Stepaniak, 2003: Covariability of components of poleward atmospheric energy transports on seasonal and interannual timescales. J. Climate, 16, 3691-3705, doi:10.1175/1520-0442(2003)016<3691:COCOPA > 2.0.CO;2.

Winton, M., 2006: Amplified Arctic climate change: What does surface albedo feedback have to do with it? Geophys. Res. Lett., 33, L03701, doi:10.1029/2005GL025244.

— W. W. Anderson, T. L. Delworth, S. M. Griffies, W. J. Hurlin, and A. Rosati, 2014: Has coarse ocean resolution biased simulations of transient climate sensitivity? Geophys. Res. Lett., 41, 8522-8529, doi:10.1002/2014GL061523.

Zelinka, M. D., and D. L. Hartmann, 2012: Climate feedbacks and their implications for poleward energy flux changes in a warming climate. J. Climate, 25, 608-624, doi:10.1175/ JCLI-D-11-00096.1.

Zurita-Gotor, P., and R. S. Lindzen, 2007: Theories of baroclinic adjustment and eddy equilibration. The Global Circulation of the Atmosphere, T. Schneider and A. H. Sobel, Eds., Princeton University Press, 22-46. 